\title{
Induced magic labeling of some graphs
}

\author{
K.B. Libeeshkumar ${ }^{1 *}$ and V. Anil Kumar ${ }^{2}$
}

\begin{abstract}
Let $G=(V, E)$ be a graph and let $(A,+)$ be an Abelian group with identity element0. Let $f: V \rightarrow A$ be a vertex labeling and $f^{*}: E \rightarrow A$ be the induced labeling of $f$, defined by $f^{*}\left(v_{1} v_{2}\right)=f\left(v_{1}\right)+f\left(v_{2}\right)$ for all $v_{1} v_{2} \in E$. Then $f^{*}$ again induces a labeling say $f^{* *}: V \rightarrow A$ defined by $f^{* *}(v)=\sum_{v v_{1} \in E} f^{*}\left(v v_{1}\right)$. A graph $G=(V, E)$ is said to be an Induced $A$-Magic Graph (IAMG) if there exists a non zero labeling $f: V \rightarrow A$ such that $f \equiv f^{* *}$. The function $f$, so obtained is called an Induced $A$-Magic Labeling (IAML) of $G$ and a graph which has no such Induced Magic Labeling is called a Non-induced magic graph. In this paper we discuss the existence of Induced Magic Labeling of some special graphs like $P_{n}, C_{n}, K_{n}$ and $K_{m, n}$.
\end{abstract}

\section{Keywords}

Induced A-Magic Labeling of Graphs, Induced A-Magic graphs.

\section{AMS Subject Classification}

05C78, 05C25.

1,2 Department of Mathematics University of Calicut, Malappuram, Kerala-670007, India.

*Corresponding author: ${ }^{1}$ libeesh123@gmail.com; ${ }^{2}$ anil@uoc.ac.in

Article History: Received 11 October 2019; Accepted 27 December 2020

(C)2020 MJM.

\section{Contents}

Introduction 59 References 59 61

\section{Introduction}

This paper deals with only finite, un directed simple and connected graphs. We refer [3] for the phrasing and standard notations related to graph theory. A graph is a pair $G=(V, E)$, where $V, E$ are the vertex set and edge set respectively. The degree of a vertex $v$ in $G$ is the number of edges incident at $v$ and it is denoted as $\operatorname{deg}(v)$. Let $(A,+)$ be an Abelian group with identity element0. Let $f: V \rightarrow A$ be a vertex labeling and $f^{*}: E \rightarrow A$ be the induced edge labeling of $f$, defined by $f^{*}\left(v_{1} v_{2}\right)=f\left(v_{1}\right)+f\left(v_{2}\right)$ for all $v_{1} v_{2} \in E$. Then $f^{*}$ again induces a vertex labeling say $f^{* *}: V \rightarrow A$ defined by $f^{* *}(v)=\sum_{v v_{1} \in E} f^{*}\left(v v_{1}\right)$. A graph $G=(V, E)$ is said to be an an Induced $A$-Magic Graph (IAMG) if there exists a non zero labeling $f: V \rightarrow A$ such that $f \equiv f^{* *}$. The function $f$, so obtained is called an Induced $A$-Magic Labeling (IAML) of $G$ and a graph which has no such Induced Magic Labeling is called a Non-induced magic graph. If an induced magic labeling $f$ where $f(v)=k$ for all verex $v$ in $G$, then $f$ is called $k$-induced magic labeling of $G$ and $G$,a $k$-induced magic graph.This paper discuss some special Induced magic graphs that belongs to the following sets:

(i) $\Gamma(A):=$ Set of all induced $A$-magic graphs.

(ii) $\Gamma(A, f):=$ Set of all induced $A$-magic graphs with IAML $f$.

(iii) $\Gamma_{k}(A):=$ Set of all induced $A$-magic graphs with kinduced magic labeling.

\section{Main Results}

Lemma 2.1. Let $G=(V, E)$ be a graph and $f$ is an IAML of $G$. If $v_{1} \in V$ is a pendant vertex adjacent to $v \in V$, then $f\left(v_{1}\right)=0$.

Proof. Let $f$ be an IAML of a graph $G$ and $v_{1}$ be a pendant vertex adjacent to $v$. Then $f^{*}\left(v v_{1}\right)=f(v)+f\left(v_{1}\right)$ and $v_{1}$ is a pendant vertex implies that $f^{* *}\left(v_{1}\right)=f(v)+f\left(v_{1}\right)$. Also $f$ is an induced magic labeling of $G$ implies that $f\left(v_{1}\right)=$ $f^{* *}\left(v_{1}\right)=f(v)+f\left(v_{1}\right)$. Thus $f(v)=0$.

Corollary 2.2. If $G$ has a pendant vertex, then $G \notin \Gamma_{k}(A)$ for any Abelian group $A$.

Proof. Proof is indisputable from the lemma 2.1. 
Lemma 2.3. Let $f$ be an IAML of a graph $G$ and wuvz be $a$ path in $G$ with $w$ and $z$ are pendant vertices in $G$, then $f^{*}(u v)=0$.

Proof. Suppose $f$ is an IAML of a graph $G=(V, E)$ and $w u v z$ is any path in $G$ with $w$ and $z$ are pendant vertices. Then by the lemma 2.1, we have $f(u)=0=f(v)$. Hence $f^{*}(u v)=0$.

Theorem 2.4. Let $f$ be a vertex labeling of a graph $G$. Then $f$ is an IAML of $G$, if and only if $[\operatorname{deg}(u)-1] f(u)+\sum f(v)=0$, for any vertex $u \in V(G)$, where the summation is taken over all the vertices $v$ which are adjacent to $u$.

Proof. Let $f$ be an IAML of $G$ and $u$ be a vertex in $G$ with $\operatorname{deg}(u)=m$. Let $v_{1}, v_{2}, v_{3}, \ldots, v_{m}$ be those vertices adjacent to $u$ in $G$. Now $f$ is an IAML if and only if $f(u)=f^{* *}(u)=$ $f^{*}\left(u v_{1}\right)+f^{*}\left(u v_{2}\right)+f^{*}\left(u v_{3}\right)+\cdots+f^{*}\left(u v_{m}\right)=m f(u)+f\left(v_{1}\right)$ $+f\left(v_{2}\right)+f\left(v_{3}\right)+\cdots+f\left(v_{m}\right)$.

That is if and only if $(m-1) f(u)+\sum f(v)=0$, where $v$ is adjacent to $u$.

Theorem 2.5. $P_{n} \in \Gamma(A)$ if and only if $n$ is a multiple of 3 .

Proof. Suppose $n=3 m$, for some integer $m$. Let $P_{n}$ be the path with vertex set $V=\left\{v_{1}, v_{2}, v_{3}, \ldots, v_{n-1}, v_{n}\right\}$. For any $a \neq$ 0 in $A$, define $f: V \rightarrow A$ as :

$$
f\left(v_{i}\right)=\left\{\begin{array}{lll}
a & \text { if } & i=1,4,7, \cdots, 3 m-2 \\
0 & \text { if } \quad i=2,5,8, \cdots, 3 m-1 \\
a^{-1} & \text { if } \quad i=3,6,9, \cdots, 3 m
\end{array}\right.
$$

Then, $f$ is an IAML of $P_{n}$. Conversely suppose $n$ is not a multiple of 3 , then $n=3 m+1$ or $n=3 m+2$ for some positive integer $m$. Let $f: V \rightarrow A$ be a vertex labeling function with $f \equiv$ $f^{* *}$.Then for $1 \leq k \leq n-3$ and any path $v_{k} v_{k+1} v_{k+2} v_{k+3}$ in $P_{n}$, we have $f\left(v_{k+1}\right)=f^{* *}\left(v_{k+1}\right)$ implies that $f\left(v_{k}\right)+f\left(v_{k+1}\right)+$ $f\left(v_{k+2}\right)=0$. Also $f\left(v_{k+2}\right)=f^{* *}\left(v_{k+2}\right)$ implies that $f\left(v_{k+1}\right)+$ $f\left(v_{k+2}\right)+f\left(v_{k+3}\right)=0$. Therefore we should have $f\left(v_{k}\right)=$ $f\left(v_{k+3}\right)$. Let us deal with the following cases:

Case 1: $n=3 m+1$

In this context, from the above discussion we have, $0=f\left(v_{2}\right)=f\left(v_{5}\right)=f\left(v_{8}\right)=\cdots=f\left(v_{3 m-1}\right)=f\left(v_{n-2}\right)$ and $0=f\left(v_{n-1}\right)=f\left(v_{n-4}\right)=\cdots=f\left(v_{6}\right)=f\left(v_{3}\right)=0$. Thus $f\left(v_{3}\right)=0$ and $f\left(v_{1}\right)+f\left(v_{3}\right)=0$ implies that $f\left(v_{1}\right)=0$, which again implies that $0=f\left(v_{1}\right)=f\left(v_{4}\right)=$ $f\left(v_{7}\right)=\cdots=f\left(v_{3 m+1}\right)=f\left(v_{n}.\right)$ Hence $f \equiv 0$, Therefore $f$ is not an IAML.

Case 2 : $n=3 m+2$

In this context from the above discussion we have, $0=$ $f\left(v_{2}\right)=f\left(v_{5}\right)=f\left(v_{8}\right)=\cdots=f\left(v_{3 m+2}\right)=f\left(v_{n}\right)$ and $0=f\left(v_{n-1}\right)=f\left(v_{n-4}\right)=\cdots=f\left(v_{4}\right)=f\left(v_{1}\right)$. Thus $f\left(v_{1}\right)=0$ and $f\left(v_{1}\right)+f\left(v_{3}\right)=0$ implies that $f\left(v_{3}\right)=$ 0 , which implies $0=f\left(v_{3}\right)=f\left(v_{6}\right)=f\left(v_{9}\right)=\cdots=$ $f\left(v_{3 m}\right)=f\left(v_{n-2}\right.$. $)$ Hence $f \equiv 0$. Therefore, $f$ is not an IAML.
Hence if $n$ is not a multiple of a 3, then $P_{n} \notin \Gamma(A)$

Theorem 2.6. Let $\left\{v_{1}, v_{2}, v_{3} \cdots, v_{n-1}, v_{n}=v_{0}\right\}$ be the vertex set of $C_{n}$. Then for any path $v_{k-1} v_{k} v_{(k+1) \bmod n}, f$ is an IAML of $C_{n}$ if and only if $f\left(v_{k-1}\right)+f\left(v_{k}\right)+f\left(v_{(k+1) \bmod n}\right)=0$, where $1 \leq k \leq n$. Moreover any IAML $f$ of $C_{n}$ satisfies $f\left(v_{k}\right)=$ $f\left(v_{(k+3) \bmod n}\right)$ for $1 \leq k \leq n$.

Proof. For $k=1,2,3, \cdots, n$, consider the path $v_{k-1} v_{k}$ $v_{(k+1) \bmod n}$ in $C_{n}$. Observe that $f$ is an IAML of $C_{n}$ if and only if $f\left(v_{k}\right)=f^{* *}\left(v_{k}\right)$, which holds if and only if $f\left(v_{k-1}\right)+f\left(v_{k}\right)$ $+f\left(v_{(k+1) \bmod n}\right)=0$.

Also for any $0 \leq k \leq n-1$, let $v_{k} v_{k+1} v_{[(k+2) \bmod n]} v_{[(k+3) \bmod n]}$, is a path in $C_{n}$, we have $f\left(v_{k}\right)+f\left(v_{k+1}\right)+f\left(v_{(k+2) \bmod n}\right)=0$ and $f\left(v_{k+1}\right)+f\left(v_{(k+2) \bmod n}\right)+f\left(v_{(k+3) \bmod n}\right)=0$.

Thus $f\left(v_{k}\right)=f\left(v_{(k+3) \bmod n}\right)$.

Corollary 2.7. $C_{n} \in \Gamma_{k}(A)$ if and only if $O(k)=3$, where $O(k)$ denotes the order of $k$ in $A$.

Proof. Consider $C_{n}$ with $V\left(C_{n}\right)=\left\{v_{1}, v_{2}, \cdots, v_{n-1}, v_{n}=v_{0}.\right\}$. Suppose $C_{n} \in \Gamma_{k}(A)$, that is there exist an IAML $f$ of $C_{n}$ with $f\left(v_{i}\right)=k$ for $i=1,2,3, \ldots, n$.Then by theorem 2.6 we have $3 k=0$ in $A$,which implies $O(k)=3$.Conversely suppose $O(k)=3$. Then consider the vertex label $f\left(v_{i}\right)=k$ for $i=1,2,3, \cdots, n$. Since $f\left(v_{i}\right)=k$ for all $i$ and $O(k)=3$, we have, $f^{*}\left(v_{i} v_{i+1}\right)=2 k$ for all $i$, and which implies $f^{* *}\left(v_{i}\right)=$ $f^{*}\left(v_{i} v_{i+1}\right)+f^{*}\left(v_{i-1} v_{i}\right)=4 k=k=f\left(v_{i}\right)$, for all $i$. Thus $f$ is an IAML of $C_{n}$, that is $C_{n} \in \Gamma_{k}(A)$. Hence the proof.

Corollary 2.8. $C_{n}$ has a non-constant IAML if and only if $n$ is a multiple of 3 .

Proof. Consider $C_{n}$ with vertex set $\left\{v_{1}, v_{2}, \ldots, v_{n-1}, v_{n}=v_{0} \cdot\right\}$. Suppose $n=3 k$, for some integer $k$. Let $a, b, c$ be any three distinct elements in $A$, such that $a+b+c=0$, then define $f: V\left(C_{n}\right) \rightarrow A$ as follows:

$$
f\left(v_{i}\right)=\left\{\begin{array}{lll}
a & \text { if } & i=1,4,7, \cdots, 3 k-2 \\
b & \text { if } & i=2,5,8, \cdots, 3 k-1 \\
c & \text { if } & i=3,6,9, \cdots, 3 k
\end{array}\right.
$$

Then clearly $f$ is a non constant IAML of $C_{n}$. Conversely assume that $n$ is not a multiple of 3 . Then either $n=3 k+1$ or $3 k+2$ for some integer $k$. Let $f$ be an IAML of $C_{n}$ and $f\left(v_{1}\right)=w$.

Case 1: $n=3 k+1$

In this context, by the theorem 2.6 we have:

$w=f\left(v_{1}\right)=f\left(v_{4}\right)=f\left(v_{7}\right)=\cdots=f\left(v_{3 k+1}\right)=f\left(v_{n}\right)=$ $f\left(v_{3}\right)=f\left(v_{6}\right)=f\left(v_{9}\right)=\cdots=f\left(v_{3 k}\right)=f\left(v_{2}\right)=f\left(v_{5}\right)$ $=f\left(v_{8}\right)=\cdots=f\left(v_{3 k-1}\right)$.

Thus $f\left(v_{i}\right)=w$, for $i=1,2,3, \cdots, n$.

Case 2: $n=3 k+2$

In this context, by the theorem 2.6 we have: $w=f\left(v_{1}\right)=f\left(v_{4}\right)=f\left(v_{7}\right)=\cdots=f\left(v_{3 k+1}\right)=f\left(v_{2}\right)=$ $f\left(v_{5}\right)=f\left(v_{8}\right)=\cdots=f\left(v_{3 k-1}\right)=f\left(v_{3 k+2}\right)=f\left(v_{n}\right)=$ $\underbrace{20}$ 


$$
f\left(v_{0}\right)=f\left(v_{3}\right)=f\left(v_{6}\right)=f\left(v_{9}\right)=\cdots f\left(v_{3 k}\right) \text {. }
$$

Thus in this case also $f\left(v_{i}\right)=w$, for $i=1,2,3, \cdots, n$.

Thus in either case, we have $f\left(v_{i}\right)=w$ for $i=1,2,3, \cdots, n$. Thus if $n \not \equiv 0(\bmod 3)$ then every IAML of $C_{n}$ is a constant IAML of $C_{n}$.

Theorem 2.9. The complete graph $K_{n} \in \Gamma(A, f)$ if and only if $(n-3) f\left(v_{1}\right)=(n-3) f\left(v_{2}\right)=(n-3) f\left(v_{3}\right)=\cdots=$ $(n-3) f\left(v_{n}\right)=-\left[f\left(v_{1}\right)+f\left(v_{2}\right)+f\left(v_{3}\right)+\cdots+f\left(v_{n}\right)\right]$ where $v_{1}, v_{2}, v_{3} \ldots, v_{n}$ are the vertices of $K_{n}$.

Proof. For $1 \leq i, j \leq n$, we have $f\left(v_{i}\right)=f^{* *}\left(v_{i}\right)$ holds if and only if $f\left(v_{1}\right)+f\left(v_{2}\right)+f\left(v_{3}\right)+\cdots f\left(v_{i-1}\right)+(n-2) f\left(v_{i}\right)+$ $f\left(v_{i+1}\right)+\cdots+f\left(v_{n}\right)=0$, similarly the condition $f\left(v_{j}\right)=$ $f^{* *}\left(v_{j}\right)$ is equivalent to the condition $f\left(v_{1}\right)+f\left(v_{2}\right)+f\left(v_{3}\right)+$ $\cdots f\left(v_{j-1}\right)+(n-2) f\left(v_{j}\right)+f\left(v_{j+1}\right)+\cdots+f\left(v_{n}\right)=0$. Thus we have $\mathrm{f}$ is an IAML if and only if $(n-3) f\left(v_{i}\right)=$ $(n-3) f\left(v_{j}\right)=-\left[f\left(v_{1}\right)+f\left(v_{2}\right)+f\left(v_{3}\right)+\cdots+f\left(v_{n}\right)\right]$, for $1 \leq i, j \leq n$. Hence the proof.

Corollary 2.10. $K_{n} \in \Gamma_{k}(A)$ if and only if $O(k)$ divides $2 n-3$, where $O(k)$ denotes the order of $k$ in $A$.

Proof. Let $K_{n}$ be the complete graph with vertex set $\left\{v_{1}, v_{2}, v_{3}\right.$ $\left.\cdots, v_{n}\right\}$. We have $K_{n} \in \Gamma_{k}(A)$, means there exist an IAML $f$ with $f(v)=k$, for all $v \in V\left(K_{n}\right)$. Also by the theorem 2.9, we have $f$ is an IAML of $K_{n}$ if and only if $(n-3) f(v)=$ $-\left[f\left(v_{1}\right)+f\left(v_{2}\right)+f\left(v_{3}\right)+\cdots+f\left(v_{n}\right)\right]$, for all $v \in V\left(K_{n}\right)$.

Thus $K_{n} \in \Gamma_{k}(A)$ if and only if $(n-3) k=-n k$, that is if and only if $(2 n-3) k=0$, that is if and only if $O(k)$ divides $2 n-3$ in $A$. Completes the proof.

Theorem 2.11. $K_{m, n} \in \Gamma_{k}(A)$ if and only if $O(k)$ divides $2 m-$ 1 and $O(k)$ divides $2 n-1$, where $O(k)$ denotes the order of $k$ in $A$.

Proof. Let $V\left(K_{m, n}\right)=\left\{v_{1}, v_{2}, v_{3}, \cdots, v_{m}, u_{1}, u_{2}, u_{3}, \cdots, u_{n}\right\}$ with each $\left(v_{i} u_{j}\right) \in E\left(K_{m, n}\right)$, for $1 \leq i \leq m, 1 \leq j \leq n$. Suppose $K_{m, n} \in \Gamma_{k}(A)$, then we have there exist an IAML $f$ with $f\left(v_{i} u_{j}\right)=k$, for $1 \leq i \leq m, 1 \leq j \leq n$. Now $f$ is an IAML of $K_{m, n}$ implies $k=f\left(v_{1}\right)=f^{* *}\left(v_{1}\right)=2 n k$, since $f^{*}\left(v_{1} u_{j}\right)=2 k$ for $1 \leq j \leq n$, that is $(2 n-1) k=0$ in $A$, which implies $O(k)$ divides $2 n-1$. similarly by considering the equation $f\left(u_{1}\right)=f^{* *}\left(u_{1}\right)$ we get $k=f\left(u_{1}\right)=f^{* *}\left(u_{1}\right)=2 m k$, that is $(2 m-1) k=0$ in $A$, which implies $O(k)$ divides $2 m-1$. Conversely suppose that $O(k)$ divides $2 m-1$ and $O(k)$ divides $2 n-1$. Consider the vertex label $f\left(v_{i}\right)=k=f\left(u_{j}\right)$, for $v_{i}, u_{j} \in V\left(K_{m, n}\right), 1 \leq i \leq m, 1 \leq j \leq n$. Then $f^{*}\left(v_{i}, u_{j}\right)=2 k$ for $1 \leq i \leq m, 1 \leq j \leq n$. There for $i=1,2,3, \cdots, m, f^{* *}\left(v_{i}\right)=$ $\sum_{j=1}^{n} f^{*}\left(v_{i} u_{j}\right)=2 n k=k$, since $O(k)$ divides $2 n-1$. Thus we have $f^{* *}\left(v_{i}\right)=f\left(v_{i}\right)=k$ for $i=1,2,3, \cdots, m$. In a similar way, we have $f^{* *}\left(u_{j}\right)=f\left(u_{j}\right)=k$ for $j=1,2,3, \cdots, n$. Hence we have $f=f^{* *}$, Thus we get $K_{m, n} \in \Gamma_{k}(A)$. This concludes the proof.

\section{References}

[1] R. Balakrishnan and K. Ranganathan, A Textbook of Graph Theory, Springer, 2012.

[2] F. Harary, Graph Theory, Addison-Wesley, Reading, MA, 1972.

[3] Chartrand G, Zhang P, Introduction to Graph Theory, McGraw-Hill, Boston; 2005.

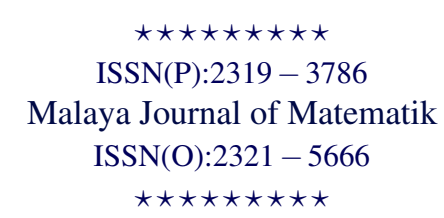

ISSN(O):2321-5666 